\title{
Self-extubation in ICU patients
}

\author{
Kavitha Selvan BS, Hawa Edriss MD, Mark Sigler MD, Jim Tseng BA
}

\section{Frequency}

The incidence of unplanned extubations per 100 intubated patients, which includes both self-extubation and accidental extubation, ranges from 0.514.2 patients. In a retrospective study of 273 patients admitted to the ICU and requiring intubation, Singh reported a $12.4 \%$ incidence of self-extubation. ${ }^{1}$ When calculated per 100 intubation days, the incidence of unplanned extubation is $0.3-4.0$ per 100 days. Selfextubation, defined as a deliberate action taken by the patient to remove the endotracheal tube, accounts for $68 \%-95 \%$ of all unplanned extubations. ${ }^{2}$ Accidental extubation refers to any non-deliberate action, such as coughing, tube manipulation, etc., taken by either medical personnel or the patient that results in removal of the endotracheal tube. ${ }^{2}$

\section{Circumstances}

\section{A. Patient Related}

Agitation in the intubated patient accounts for $50-74 \%$ of the unplanned extubations and is the most significant risk factor for these events. ${ }^{3}$ Patient agitation is related to numerous factors, including prolonged immobility, the inability to communicate, and nursing and respiratory procedures, and increases the rate of self-extubation by $26 \%$ compared to nonagitated patients. ${ }^{3,4}$ In addition to agitation, the patient's level of consciousness is a strong predictor for self-extubation, and incidence is higher in patients with a Glasgow Coma scale score of $9-12$ points. ${ }^{5}$

Corresponding author: Kavitha Selvan, BS Contact Information: Kavitha.selvan@ttuhsc.edu DOI: 10.12746/swrccc2014.0208.100
Additional factors that correlate with a higher frequency of self-extubation include male gender $(67 \%)$, sedation given on an "as needed" basis rather than as a continuous infusion, and a current history of smoking. ${ }^{6}$ Self-extubation is more common in surgical patients, burn patients, and older patients. In a retrospective study conducted by Tung, the average age of patients who self-extubated was 65.3 years. ${ }^{7}$ Patients between ages $30-50$ account for $47 \%$ of self-extubation episodes; $58.9 \%$ of episodes occur in surgical patients. ${ }^{1}$ Self-extubation also occurs more frequently in patients with certain conditions, such as chronic obstructive pulmonary disease, adult respiratory distress syndrome, or cardiac disease. ${ }^{6}$ This increased frequency could, however, simply reflect the longer intubation periods and weaning in these patient groups. ${ }^{2}$

\section{B. NuRse RELATED}

Nursing care is an important factor that contributes to a patient's likelihood to self-extubate, and the attending nurse's absence from the bedside is the most important predictor. This is closely followed by decreased patient surveillance and a low nurse to patient ratio. The optimal ratio to decrease the incidence of self-extubation is probably one to one, and while this may not be feasible, patients with a high risk of self-extubation should be allocated more supervision. ${ }^{3}$ In addition, less experienced nurses are more likely to encounter self-extubation; staffing with registered nurses has significantly reduced the risk of selfextubation. ${ }^{8}$ It has been shown that patients under the care of an ICU nurse with $>4$ years of experience have a $2.6 \%$ lower incidence of self-extubations. ${ }^{3}$

The incidence of self-extubation is higher during the night shift $(76 \%)$, which could reflect a higher risk for patient delirium at night or decreased patient 
surveillance. ${ }^{5}$ The effect of decreased patient surveillance on self-extubation is also demonstrated by the higher frequency of self-extubations occurring within the hour before and after shift changes, when patients are often monitored less. Self-extubation during shift changes between 7:00 am and 8:30 am and 7:00 pm and 8:30 pm accounted for almost $50 \%$ of the self-extubations in a tertiary care ICU over a one year period. ${ }^{1}$

\section{Outcomes}

Self-extubation has the potential to damage the larynx and cause severe airway complications due to removing the tube with the cuff still inflated. Hypotension, arrhythmias, bronchospasm, aspiration, and laryngeal bleeding or edema can also occur. In addition, up to $20 \%$ of patients have a difficult re-intubation following self-extubation. ${ }^{2}$

However, self-extubation often occurs in patients who are ready for elective extubation within the next few hours. Patients who self-extubate within 72 hours of a planned extubation require re-intubation in only $14 \%$ of cases. Therefore, in the majority of patients who do not require re-intubation, self-extubation can actually decrease the length of intubation and weaning. 2,3,9

The complications of self-extubation are seen most often in patients requiring re-intubation, which occurs within an hour of self-extubation in $85 \%-90 \%$ of cases. ${ }^{2}$ Risk factors for re-intubation include a higher pre-extubation fraction of inspired oxygen $\left(\mathrm{FiO}_{2}\right)$, a lower ratio of arterial oxygen tension to inspired oxygen concentration $\left(\mathrm{PaO}_{2} / \mathrm{FiO}_{2}\right)$, assist/control ventilation mode, female gender, decreased mental status related to sedation or illness, organ dysfunction, infection, increased pulmonary secretions, tachycardia, temperature greater than $37.5^{\circ} \mathrm{C}, \mathrm{pH}$ greater than 7.45 , and poor pulmonary compliance. . $^{2,3}$ Only $45 \%$ of patients who self-extubate require re-intubation; however, these patients have a sevenfold higher ICU or hospital mortality rate compared to successfully self-extubated patients. ${ }^{2,5}$ In addition, patients who are re-intubated are more likely to have nosocomial ventilator-associated pneumonia. ${ }^{2}$

\section{Prevention}

Physical restraints have been historically used to prevent self-extubation; however, according to Tung, restraint use is actually associated with selfextubation. He reported that $65 \%$ of patients who self-extubated were restrained either at the time of self-extubation or within 24 hours of self-extubation. ${ }^{7}$ Restraints are often used with agitated or delirious patients, whose risk for self-extubation is already increased. A quality improvement initiative increased restraint use in agitated patients from 58\% to $90 \%$ over four years and saw a $6 \%$ decrease in the number of self-extubations. It is important to note, howev$\mathrm{er}$, that restraints can worsen agitation or delirium and increase the risk for self-extubation. It is likely that the reduction in self-extubation was due to an increase in sedation, which ranged from $39 \%$ to $77 \%$ during the four years. ${ }^{10}$ The head-up position, with the bed elevated at 30 degrees, increases the risk for self-extubation by allowing the patient's hands to be in closer to the endotracheal tube. While this position may help prevent aspiration, it also promotes self-extubation and should be limited in some patients. ${ }^{2}$

The lack of an appropriate sedation level is the most common factor contributing to self-extubation in the ICU. Achieving optimal sedation can be difficult, as titration of appropriate sedation depends on both the hemodynamic and neurological status of the patient and differs from patient to patient. The goal is for the patient to tolerate the endotracheal tube, while at the same time maintaining consciousness. Patients with low sedation levels have a higher incidence of self-extubation, possibly explained by a lack of tolerance for the endotracheal tube and increased ventilator-patient asynchrony. ${ }^{1}$ Use of the Modified Ramsay Sedation Scale protocol has been shown to decrease self-extubation from $7 \%$ to $3 \%$ within one year due to appropriate management of patient agitation. ${ }^{11}$ However, it is equally important to avoid over sedation in mechanically ventilated patients, since this has been associated with prolonged mechanical ventilation, increased ICU length of stay, and an increased incidence of nosocomial infection. ${ }^{3}$ Use of a nurse-driven sedation protocol can help prevent over sedation and significantly reduce the frequency of self-extubation. 
Nursing compliance with a weaning protocol also lowers the risk of self-extubation in medical ICU patients. In Jarachovic's study, $82 \%$ of patients who self-extubated were not on a weaning protocol; this demonstrates the need for a standardized weaning protocol. 12 Weaning protocols reduce the overall duration of mechanical ventilation, re-intubation rates, and nosocomial infections. ${ }^{3}$

The Self-Extubation Risk Assessment Tool (SERAT) was developed to identify patients at a high risk for self-extubation (Figure 1). It is based on the Bloomsbury Sedation Score (which is similar to the Richmond agitation scale) and the Glasgow Coma Scale and has $100 \%$ sensitivity and $79 \%$ specificity in identifying patients at risk for self-extubation. ${ }^{3}$ The SERAT tool predicts the risk for self-extubation with the highest accuracy when the Bloomsbury Sedation Score-Glasgow Coma Scale scores fall within the dark grey zone (top right zone). The risk for self-extubation can be predicted with the highest sensitivity when the scores falls within the light grey zone (middle zone). ${ }^{13}$ This tool indicates that higher Glasgow Coma scale scores and lower sedation scores both influence the risk for self-extubation. Patients identified by this tool as at risk should be monitored more closely by nursing personnel to prevent self-extubation.

\section{ICU QuaLity ImProvement}

Monitoring self-extubation rates in ICUs provides a good ICU care monitoring tool. An increased number of self-extubations requiring re-intubation indicates a need to review nursing and sedation protocols. An increased number of self-extubations not requiring re-intubation indicates a need to review weaning protocols. Tracking the number per month provides a trend that might lead to a root-cause analysis if the number goes up.

\section{Key Points}

1. The most common risk factor for self-extubation is inadequate sedation that leads to patient agitation. Optimal sedation can be difficult to achieve, but the use of sedation protocols can reduce self-extubation rates.

2. Self-extubation is more common in surgical patients, who account for $58.9 \%$ of all self-extubation cases. ${ }^{1}$ These patients should be monitored closely by experienced nurses who can help decrease the rate of self-extubation.

3. Self-extubations often occur in patients scheduled for elective extubation within a few hours, suggesting that health care personnel need to better manage weaning and extubation timing.

4. The frequency of self-extubation is higher during the hour before and after nursing shift changes, and this factor was relevant in almost $50 \%$ of selfextubations in a tertiary care ICU over a one year period. ${ }^{1}$ Adopting a practice of increased patient surveillance during shift changes could help significantly decrease the incidence of self-extubation.

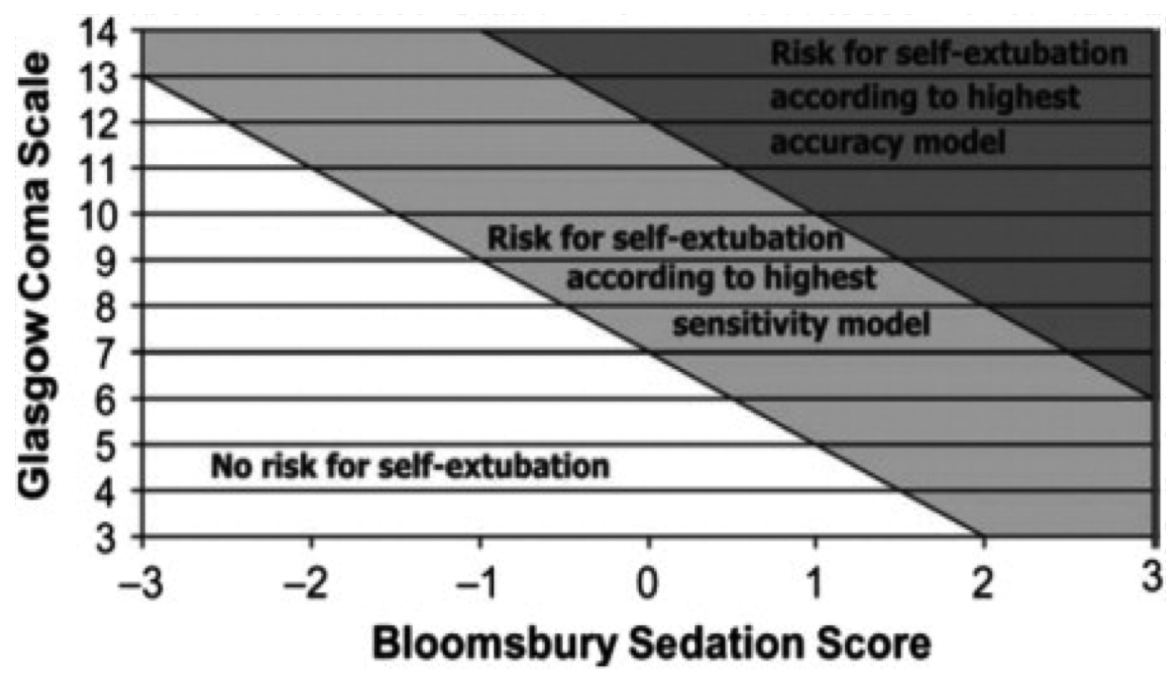


Author Affiliation: Kavitha Selvan and Jim Tseng are medical students at TTUHSC. Hawa Edriss is a resident in internal medicine and Mark Sigler is a fellow in pulmonary and critical care medicine at TTUHSC.

Received: 07/09/2014

Accepted: 10/02/2014

Reviewers: Isham Huizar MD, Randall Rosenblatt MD

Published electronically: 10/15/2014

Conflict of Interest Disclosures: None dation on the incidence of self-extubation. Am J Med Qual 2000; 15(5): 221-225.

11. Powers J. A sedation protocol for preventing patient selfextubation. Dimensions Crit Care Nursing 1999; 18(2): 3034.

12. Jarachovic $M$, Mason $M$, Kerber $K$, McNett $M$. The role of standardized protocols in unplanned extubations in a medical intensive care unit. Am J Crit Care 2011; 20(4): 304-312.

13. Moons P, Boriau M, Ferdinande P. Self-extubation risk assessment tool: predictive validity in a real-life setting. Nurs Crit Care 2008; 13(6): 310-4.

\section{REFERENCES}

1. Singh PM, Rewari V, Chandralekha, Arora MK, Trikha A. A retrospective analysis of determinants of self-extubation in a tertiary care intensive care unit. J Emerg Trauma Shock 2013; 6(4): 241-5.

2. Kiekkas P, Aretha D, Panteli E, Baltopoulos GI, Filos, KS. Unplanned extubation in critically ill adults: clinical review. Nursing Crit Care 2012; 18(3): 123-34.

3. King JN, Elliott VA. Self/unplanned extubation: safety, surveillance, and monitoring of the mechanically ventilated patient. Crit Care Nurs Clin North Am 2012; 24(3): 469-79. 4. Woods JC, Mion LC, Connor JT, Viray F, Jahan L, Huber C, McHugh R, Gonzales JP, Stoller JK, Arroliga AC. Severe agitation among ventilated medical intensive care unit patients: frequency, characteristics and outcomes. Intensive Care Med 2004; 30(6): 1066-72.

5. Chang LC, Liu PF, Huang YL, Yang SS, Chang WY. Risk factors associated with unplanned endotracheal self-extubation of hospitalized intubated patients. Applied Nursing Research 2011; 24(3): 188-192.

6. Balon JA. Common factors of spontaneous self-extubation in a critical care setting. International J Trauma Nursing 2001; 7(3): 93-99.

7. Tung A, Tadimeti L, Caruana-Montaldo B, Atkins PM, Mion LC, Palmer RM, Slomka J, Mendelson W. The relationship of sedation to deliberate self-extubation. J Clin Anesthesia 2001; 13(1): 24-29.

8. Kane RL, Shamliyan TA, Mueller C, Duval S, Wilt TJ. The association of registered nurse staging levels and patient outcomes: systemic review and meta-analysis. Med Care 2007; 45(12): 1195-1204.

9. Krinsley JS, Reddy PK, Iqbal A. What is the optimal rate of failed extubation? Crit Care 2012; 16(1): 111.

10. Frezza EE, Carleton GL, Valenziano CP. A quality improvement and risk management initiative for surgical ICU patients: a study of the effects of physical restraints and se- 